\title{
OFDM CHANNEL ESTIMATION IN THE PRESENCE OF PHASE NOISE AND FREQUENCY OFFSET BY PARTICLE FILTERING
}

\author{
François Septier $^{1}$, Yves Delignon ${ }^{2}$, Atika Menhaj-Rivenq ${ }^{1}$, Christelle Garnier ${ }^{2}$ \\ 1 - IEMN-DOAE UMR 8520, U.V.H.C. Le Mont Houy, 59313 Valenciennes Cedex 9, France \\ 2 - GET/INT/Telecom Lille 1, 59658 Villeneuve d'Ascq, France
}

\begin{abstract}
In this paper, we address the problem of OFDM channel estimation in the presence of phase noise (PHN) and carrier frequency offset (CFO). For OFDM systems, PHN and CFO cause two effects: the common phase error (CPE) and the intercarrier interference (ICI) which severely degrade the accuracy of the channel estimate. In literature, several algorithms have been proposed to solve this problem. Here, we propose the joint estimation of channel impulse response (CIR), CFO and PHN with no prior statistical knowledge of PHN and SNR. The proposed approach uses a training OFDM symbol to track and estimate these many unknowns in the time domain by particle filtering. The particle filter is efficiently implemented by combining the principles of the Rao-Blackwellization technique and the hybrid importance function which encompasses the advantages of both the optimal and the prior importance functions. Simulation results are provided to illustrate the effectiveness of the proposed algorithm.
\end{abstract}

Index Terms - Communication systems, Estimation, Phase noise, Monte Carlo methods.

\section{INTRODUCTION}

Orthogonal frequency division multiplexing (OFDM) is known to be an efficient technique for high-rate transmission that can overcome the intersymbol interference (ISI) resulting from the time dispersion of multipath fading channels. It has been adopted as the transmission method of many standards in wireline and wireless communications, such as digital subscriber lines (DSL), digital and audio broadcasting (DAB/DVB), wireless area networks (IEEE 802.11) and broadband wireless access (IEEE 802.16).

Unfortunately, OFDM systems are very sensitive to phase noise (PHN) and to carrier frequency offset (CFO) respectively caused by oscillator imperfections and Doppler shifts [1-3]. Indeed, these phase distortions destroy the orthogonality of the OFDM subcarriers and lead both to rotation of every subcarrier by a random phase, called common phase error (CPE), and to intercarrier interference (ICI).

Many approaches have been proposed to estimate and compensate PHN [4-6] or both CFO and PHN [7]. Nevertheless in these algorithms, the channel impulse response (CIR) is assumed to be known prior to phase distortion suppression. Channel estimation in the presence of both PHN and CFO has been adressed in [8,9]. In [8], the authors focus principally on both channel and CFO estimation. Recently, in [9], a maximum a posteriori estimator of channel response, PHN and CFO incorporating prior knowledge of SNR and PHN statistics is proposed through the maximization of a complete likelihood function.
In this paper, we consider the channel estimation problem in the presence of PHN and CFO with no a priori knowledge of operating SNR and PHN statistics. To estimate these unknown states with a single OFDM symbol, we propose a marginalized particle filter based on the hybrid importance function. To allow the use of this hybrid importance function, we derive an approximation of the optimal importance function for sampling the PHN distortions.

The paper is organized as follows. The OFDM system model and the PHN model are introduced in Section 2 leading to the dynamic state space representation. Section 3 is devoted to the particle filtering. We describe the proposed marginalized particle filter for joint channel, CFO, PHN and a priori statistics estimation. The simulation results are provided in Section 4 showing the effectiveness of the proposed algorithm. Finally, conclusions are given in Section 5.

\section{SYSTEM MODEL}

\subsection{Signal Model}

We consider an OFDM system with $N$ subcarriers. The transmitted OFDM signal $s_{k}$ is generated via an inverse FFT operation applied on the subcarrier symbols $d_{k}$. To prevent intersymbol interference (ISI), a cyclic prefix of length $N_{c p}$ is placed in front of the useful part of duration $T$ of the signal.

We assume a slow fading frequency-selective channel with $L$ paths. The CIR remains constant during one transmission packet including several OFDM symbols. Assuming perfect timing synchronization and $L \leq N_{c p}$, the complex baseband received OFDM signal can be written, after removal of the cyclic prefix :

$$
r_{k}=e^{j\left(\theta_{k}+2 \pi k \epsilon / N\right)} \sum_{l=0}^{L-1} h_{l} s_{k-l}+b_{k}
$$

where $k$ denotes the $k$-th sample of the OFDM symbol and $\left\{h_{l}\right\}_{l=0}^{L-1}$, $\left\{s_{k}\right\}_{k=0}^{N-1},\left\{\theta_{k}\right\}_{k=0}^{N-1}$ and $\left\{b_{k}\right\}_{k=0}^{N-1}$ denote respectively the CIR, the known transmitted signal, the PHN and a circular zero mean gaussian white noise with power $\sigma_{b}^{2} . \epsilon=\Delta f T$ is the normalized carrier frequency offset (CFO). Equation (1) can be written in the matrix form as :

$$
r_{k}=e^{j\left(\theta_{k}+2 \pi k \epsilon / N\right)} \mathbf{S}_{k} \mathbf{h}+b_{k}
$$

where $\mathbf{S}_{k}=\left[\begin{array}{lll}s_{k} & \cdots & s_{k-L+1}\end{array}\right]$ is the transmitted OFDM signal vector and $\mathbf{h}=\left[\begin{array}{lll}h_{0} & \cdots & h_{L-1}\end{array}\right]^{T}$ is the CIR vector.

\subsection{Phase Noise Model}

In a baseband complex equivalent form, the carrier delivered by the noisy oscillator can be modeled as $p(t)=\exp (j \theta(t))$, where the 
phase distortion $\theta(t)$ is a Brownian process [1,2]. Its power spectral density has a Lorentzian shape controlled by the parameter $\beta$ representing the two-sided $3 \mathrm{~dB}$ bandwidth. This model produces a $1 / f^{2}$ type noise power behavior that agrees with experimental measurements carried out on real RF oscillators. The phase noise rate is characterized by the bandwidth $\beta$ normalized with respect to the OFDM symbol rate $1 / T$, namely by the parameter $\beta T$. In a discrete form, the phase distortion can be written as :

$$
\theta_{k}=\theta_{k-1}+v_{k}
$$

where $v_{k}$ is a zero mean gaussian variable with variance $\sigma_{v}^{2}=2 \pi \beta T / N$.

\subsection{Dynamic State-Space Model}

This paper focuses on accurate estimation of CIR, CFO and PHN from a single OFDM symbol. At the receiver, the transmitted OFDM signal $\left\{s_{k}\right\}_{k=0}^{N-1}$ is perfectly known. However, both the instantaneous PHN power $\sigma_{v}^{2}$ and the AWGN power $\sigma_{b}^{2}$ are assumed unknown. The mathematical foundation of our solution is the Bayesian theory. This theory requires a dynamic state-space (DSS) modeling both the observation and hidden process.

By combining (3) and (2) and by using the fact that one dynamic state $\theta_{k}$ and four static parameters $\left(\mathbf{h}, \epsilon, \sigma_{v}^{2}\right.$ and $\sigma_{b}^{2}$ ) are unknown, we obtain the following dynamic state-space model :

$$
\underline{\text { State equations : }\{}\left\{\begin{array}{l}
\theta_{k}=\theta_{k-1}+v_{k} \\
\epsilon_{k}=\epsilon_{k-1}, \sigma_{v, k}^{2}=\sigma_{v, k-1}^{2}, \sigma_{b, k}^{2}=\sigma_{b, k-1}^{2}
\end{array}\right.
$$

$$
\text { Measurement Equation : } \quad r_{k}=e^{j\left(\theta_{k}+2 \pi k \epsilon_{k} / N\right)} \mathbf{S}_{k} \mathbf{h}+b_{k}
$$

Let us introduce the state vector $x_{k}$ defined as $x_{k}=\left[\mathbf{h}, \theta_{k}, \xi_{k}\right]$ with $\xi_{k}$ the vector of static parameters : $\xi_{k}=\left[\epsilon_{k}, \sigma_{v, k}^{2}, \sigma_{b, k}^{2}\right]$. Time index $k$ are inserted on static parameters $\xi$ and $\epsilon$ in order to clearly distinguish parameter estimation at the $k$-th index. Our main objective is to estimate $x_{k}$ using the a posteriori probability density function (pdf) $p\left(x_{0: k} \mid r_{0: k}\right)$. Unfortunately, this pdf is analytically intractable, so we propose a numerical approximation via particle filtering [10].

Remark: With this joint estimation, it can be shown that the phase distortions can be accurately estimated, but with a constant gap $\Phi$ from the real phase distortions. This rotation can be estimated during the data detection step using pilot symbols [9].

\section{PARTICLE FILTERING}

\subsection{Introduction}

Particle filtering is a sequential Monte Carlo sampling method built on the Bayesian paradigm [10]. From the Bayesian theory, at sample $k$, the posterior distribution $p\left(x_{0: k} \mid r_{0: k}\right)$ is the main entity of interest. However, due to the nonlinearity of the measurement equation (5), its analytical expression is not tractable. Alternatively, particle filtering can be applied to approximate this pdf by stochastic samples generated using a sequential importance sampling strategy.

Particle filtering is an extension of the sequential Monte Carlo methodology [10]. It consists in recursively estimating the required posterior density function $p\left(x_{0: k} \mid r_{0: k}\right)$ by a set of $M$ random samples with associated weights, denoted by $\left\{x_{0: k}^{(m)}, w_{k}^{(m)}\right\}_{m=1 . . M}$ :

$$
\widehat{p}\left(x_{0: k} \mid r_{0: k}\right)=\sum_{j=1}^{M} \delta\left(x_{0: k}-x_{0: k}^{(j)}\right) \widetilde{w}_{k}^{(j)}
$$

where $x_{k}^{(j)}$ is drawn from the importance function $\pi\left(x_{k} \mid x_{0: k-1}^{(j)}, r_{0: k}\right)$, $\delta($.$) is the Dirac delta function and \widetilde{w}_{k}^{(j)}=w_{k}^{(j)} / \sum_{m=1}^{M} w_{k}^{(m)}$ is the normalized importance weight associated with the $j$-th particle.

The weights $w_{k}^{(m)}$ are updated according to the concept of importance sampling :

$$
w_{k}^{(m)} \propto \frac{p\left(r_{k} \mid x_{0: k}^{(m)}\right) p\left(x_{k}^{(m)} \mid x_{0: k-1}^{(m)}\right)}{\pi\left(x_{k}^{(m)} \mid x_{0: k-1}^{(m)}, r_{0: k}\right)} w_{k-1}^{(m)}
$$

After a few iterations, particle filtering is known to suffer from degeneracy problems. So we integrate a resampling step to select particles for new generations in proportion to the importance weights [10]. Liu and Chen [11] have introduced a measure known as the effective sample size, $N_{\text {eff }}=\left[\sum_{m=1}^{M}\left(\widetilde{w}_{k}^{(m)}\right)^{2}\right]^{-1}$, and have proposed to apply the resampling procedure whenever $N_{\text {eff }}$ is below a predefined threshold. For the resampling step, we use the Residual Resampling scheme described in [12]. This scheme outperforms the simple random sampling scheme with a small Monte Carlo variance and a favorable computational time [11].

\subsection{Particle Filter for Joint CIR, CFO and PHN Estimation}

Previously, we have seen how particle filtering can be used to obtain the posterior density function $p\left(x_{0: k} \mid r_{0: k}\right)$. In order to reduce the state dimension for the particle filter, the Rao-Blackwellization technique, also known as the marginalized particle filter [10,13,14], marginalizes out conditionally linear-Gaussian state variables from the joint posterior distribution. This strategy is shown to reduce the variance of the state estimates obtained via the particle filter [13]. This is due to the fact that the particle filter is then only used to estimate the nonlinear states, while the remaining conditional linearGaussian states are estimated using the closed-form Kalman filter. In our case, conditionally on the nonlinear state variables $\theta_{k}$ and $\xi_{k}$ the DSS model contains a linear substructure on $\mathbf{h}$, subject to gaussian noise. Using the Bayes' theorem, the posterior density function of interest can be written as :

$$
p\left(x_{0: k} \mid r_{0: k}\right)=p\left(\mathbf{h} \mid \theta_{0: k}, \xi_{0: k}, r_{0: k}\right) p\left(\theta_{0: k}, \xi_{0: k} \mid r_{0: k}\right)
$$

where $p\left(\mathbf{h} \mid \theta_{0: k}, \xi_{0: k}, r_{0: k}\right)$ is analytically tractable and can be obtained via a Kalman filter associated with each particle. Indeed, the $j$-th pdf is a multidimensional gaussian probability density function. The mean $\mathbf{h}_{k \mid k}^{(j)}$ and the covariance $\Sigma_{k \mid k}^{(j)}$ can be obtained using the Kalman filtering equations given by the time update equations :

$$
\begin{aligned}
\mathbf{h}_{k \mid k-1}^{(j)} & =\mathbf{h}_{k-1 \mid k-1}^{(j)} \\
\Sigma_{k \mid k-1}^{(j)} & =\Sigma_{k-1 \mid k-1}^{(j)}
\end{aligned}
$$

and the measurement update equations :

$$
\begin{aligned}
& \chi_{k}^{(j)}=\mathbf{S}_{k} \Sigma_{k \mid k-1}^{(j)} \mathbf{S}_{k}^{H}+\sigma_{b, k}^{2(j)} \\
& K_{k}^{(j)}=\Sigma_{k \mid k-1}^{(j)}\left(e^{j\left(\theta_{k}^{(j)}+2 \pi k \epsilon_{k}^{(j)} / N\right)} \mathbf{S}_{k}\right)^{H}\left(\chi_{k}^{(j)}\right)^{-1} \\
& \mathbf{h}_{k \mid k}^{(j)}=\mathbf{h}_{k \mid k-1}^{(j)}+K_{k}^{(j)}\left(r_{k}-e^{j\left(\theta_{k}^{(j)}+2 \pi k \epsilon_{k}^{(j)} / N\right)} \mathbf{S}_{k} \mathbf{h}_{k \mid k-1}^{(j)}\right) \\
& \Sigma_{k \mid k}^{(j)}=\Sigma_{k \mid k-1}^{(j)}-K_{k}^{(j)} e^{j\left(\theta_{k}^{(j)}+2 \pi k \epsilon_{k}^{(j)} / N\right)} \mathbf{S}_{k} \Sigma_{k \mid k-1}^{(j)}
\end{aligned}
$$

where $\chi_{k}^{(j)}$ and $K_{k}^{(j)}$ are respectively the residual covariance and the optimal Kalman gain associated with the $j$-th particle. 
In addition, the marginal posterior distribution $p\left(\theta_{0: k}, \xi_{0: k} \mid r_{0: k}\right)$ in (8) can be approximated with a particle filter as :

$$
\widehat{p}\left(\theta_{0: k}, \xi_{0: k} \mid r_{0: k}\right)=\sum_{j=1}^{M} \delta\left(\theta_{0: k}-\theta_{0: k}^{(j)} ; \xi_{0: k}-\xi_{0: k}^{(j)}\right) \widetilde{w}_{k}^{(j)}
$$

where $\delta(. ;$.$) is the two-dimensional Dirac delta function. Then, our$ objective is to generate samples from the distribution $p\left(\theta_{0: k}, \xi_{0: k} \mid r_{0: k}\right)$. The choice of the importance function is essential because it determines the efficiency as well as the complexity of the particle filtering algorithm. Here, we adopt the hybrid importance function [15], which encompasses the advantages of both the posterior and the prior importance functions. The proposed hybrid importance function is expressed as :

$$
\begin{aligned}
\pi\left(\theta_{k}, \xi_{k} \mid \theta_{0: k-1}^{(j)}, \xi_{0: k-1}^{(j)}, r_{0: k}\right)= & p\left(\theta_{k} \mid \theta_{0: k-1}^{(j)}, \xi_{0: k}^{(j)}, r_{0: k}\right) \\
& \times p\left(\xi_{k} \mid \xi_{0: k-1}^{(j)}\right) \\
= & p\left(\theta_{k} \mid \theta_{0: k-1}^{(j)}, \xi_{0: k}^{(j)}, r_{0: k}\right) \\
& \times \delta\left(\xi_{k}-\xi_{0: k-1}^{(j)}\right)
\end{aligned}
$$

where (12) is obtained using the state equations in (4). The corresponding unnormalized weights are then computed by :

$$
w_{k}^{(j)} \propto w_{k-1}^{(j)} p\left(r_{k} \mid \theta_{0: k-1}^{(j)}, \xi_{0: k}^{(j)}, r_{0: k-1}\right)
$$

Now, we discuss the sampling of $\theta_{k}$ and $\xi_{k}$ from (12). First, let's note that for $\xi_{k}$ no sampling is needed, i.e. $\xi_{k}^{(j)}=\xi_{k-1}^{(j)}$ so that the time update of $\xi_{k}^{(j)}$ is not performed and the accuracy of the final estimates greatly depends on the initial samples. To adress this problem, kernel smoothing techniques or Markov chain Monte Carlo (MCMC) moves [10] can be used during the resampling procedure. However, in our context, such methods bring no improvement.

The sampling of $\theta_{k}$ requires the analytical expression of the optimal importance function. This pdf can be rewritten as :

$$
\begin{aligned}
p\left(\theta_{k} \mid \theta_{0: k-1}^{(j)}, \xi_{0: k}^{(j)}, r_{0: k}\right) \propto \quad & p\left(r_{k} \mid \theta_{k}, \theta_{0: k-1}^{(j)}, \xi_{0: k}^{(j)}, r_{0: k-1}\right) \\
& \times p\left(\theta_{k} \mid \theta_{0: k-1}^{(j)}\right)
\end{aligned}
$$

with

$$
p\left(\theta_{k} \mid \theta_{0: k-1}^{(j)}\right)=\mathcal{N}\left(\theta_{k} ; \theta_{k-1}^{(j)}, \sigma_{v, k}^{2(j)}\right)
$$

and,

$$
p\left(r_{k} \mid \theta_{k}, \theta_{0: k-1}^{(j)}, \xi_{0: k}^{(j)}, r_{0: k-1}\right)=\mathcal{N}_{c}\left(r_{k} ; \rho_{k}^{(j)}, \chi_{k}^{(j)}\right)
$$

where $\rho_{k}^{(j)}=e^{j\left(\theta_{k}^{(j)}+2 \pi k \epsilon_{k}^{(j)} / N\right)} \mathbf{S}_{k} \mathbf{h}_{k \mid k-1}^{(j)}$ and $\chi_{k}^{(j)}$ are given by the Kalman filter associated with the $j$-th particle as described in (9)-(10). $\mathcal{N}_{c}($.$) and \mathcal{N}($.$) denote respectively the circular gaussian$ distribution and the gaussian distribution. According to (15)-(16), an analytical form for (13) remains untractable due to the double exponential in $\rho_{k}^{(j)}$. However, by linearizing the noise term $v_{k}$ in (3), the mean of (16) is approximated by :

$$
\begin{aligned}
\rho_{k}^{(j)} & =e^{j\left(\theta_{k-1}^{(j)}+v_{k}+2 \pi k \epsilon_{k}^{(j)} / N\right)} \mathbf{S}_{k} \mathbf{h}_{k \mid k-1}^{(j)} \\
& \approx\left(1+j v_{k}\right) e^{j\left(\theta_{k-1}^{(j)}+2 \pi k \epsilon_{k}^{(j)} / N\right)} \mathbf{S}_{k} \mathbf{h}_{k \mid k-1}^{(j)}
\end{aligned}
$$

This approximation holds when the phase noise rate is small and is more accurate than the usual approximation $e^{j \theta_{k}} \approx 1+j \theta_{k}$. Using
(15), (16) and (17) and after several algebraic manipulations, (14) can be simplified as :

$\begin{aligned} p\left(\theta_{k} \mid \theta_{0: k-1}^{(j)}, \xi_{0: k}^{(j)}, r_{0: k}\right) \propto & C_{k}^{(j)} e^{-\frac{1}{2 \Lambda_{k}^{(j)}}\left[\frac{\left|r_{k}-\Gamma_{k}^{(j)}\right|^{2}}{\left|\Gamma_{k}^{(j)}\right|^{2} \sigma_{v, k}^{2(j)}+\chi_{k}^{(j)}}-\left(\mu_{k}^{(j)}\right)^{2}\right]} \\ & \times \mathcal{N}\left(\theta_{k} ; \mu_{k}^{(j)}+\theta_{k-1}^{(j)}, \Lambda_{k}^{(j)}\right)\end{aligned}$

where $C_{k}^{(j)}=\sqrt{\Lambda_{k}^{(j)} /\left(2 \pi \chi_{k}^{(j)} \sigma_{v, k}^{2(j)}\right)}, \mu_{k}^{(j)}=\frac{\Im\left(\Gamma_{k}^{(j) *} r_{k}\right) \sigma_{v, k}^{2(j)}}{\left|\Gamma_{k}^{(j)}\right|^{2} \sigma_{v, k}^{2(j)}+\chi_{k}^{(j)}}$ (with $\Im($.$) the imaginary part), \Lambda_{k}^{(j)}=\frac{\chi_{k}^{(j)} \sigma_{v, k}^{2(j)}}{\left|\Gamma_{k}^{(j)}\right|^{2} \sigma_{v, k}^{2(j)}+\chi_{k}^{(j)}}$ and $\Gamma_{k}^{(j)}=$ $e^{j\left(\theta_{k-1}^{(j)}+2 \pi k \epsilon_{k}^{(j)} / N\right)} \mathbf{S}_{k} \mathbf{h}_{k \mid k-1}^{(j)}$.

The sampling distribution of $\theta_{k}$ is now identified in (18) and thus only the analytical expression to update the importance weights is missing for the implementation of the particle filter. The pdf required in (13) can be rewritten as :

$$
\begin{aligned}
p\left(r_{k} \mid \theta_{0: k-1}^{(j)}, \xi_{0: k}^{(j)}, r_{0: k-1}\right)= & \int_{\mathbb{R}} p\left(r_{k} \mid \theta_{k}, \theta_{0: k-1}^{(j)}, \xi_{0: k}^{(j)}, r_{0: k-1}\right) \\
& \times p\left(\theta_{k} \mid \theta_{0: k-1}^{(j)}\right) d \theta_{k}
\end{aligned}
$$

Using (18), it is straightforward to show that (19) can be approximated as :

$p\left(r_{k} \mid \theta_{0: k-1}^{(j)}, \xi_{0: k}^{(j)}, r_{0: k-1}\right) \approx C_{k}^{(j)} e^{-\frac{1}{2 \Lambda_{k}^{(j)}}\left[\frac{\left|r_{k}-\Gamma_{k}^{(j)}\right|^{2}}{\left|\Gamma_{k}^{(j)}\right|^{2} \sigma_{v, k}^{2(j)}+x_{k}^{(j)}}-\left(\mu_{k}^{(j)}\right)^{2}\right]}$

Each element required in the implementation of the particle filtering algorithm has been identified. Finally, the minimum mean square error (MMSE) estimate of $\mathbf{h}$ is easily calculated according to

$$
\widehat{\mathbf{h}}=\sum_{j=1}^{M} \mathbf{h}_{N-1 \mid N-1}^{(j)} \widetilde{w}_{N-1}^{(j)}
$$

The proposed marginalized particle filter algorithm is summed up in Table 1.

Table 1. Marginalized Particle Filter Algorithm

$$
\begin{aligned}
& \text { Initialization, for } m=, 0 \ldots, M \\
& \left.\left.\qquad \epsilon_{-1}^{(m)} \sim \mathcal{U}[-0.5 ; 0.5], \sigma_{v,-1}^{2(m)} \sim \mathcal{U}[0 ; 0.1], \sigma_{b,-1}^{2(m)} \sim \mathcal{U}\right] 0 ; 1\right] \\
& \qquad \mathbf{h}_{-1 \mid-1}^{(m)}=\mathbf{0}_{L \times 1}, \Sigma_{-1 \mid-1}^{(m)}=\frac{1}{L} \mathbf{I}_{L}, \widetilde{w}_{k}^{(m)}=1 / M \\
& \text { For } k=0, \ldots, N-1 \\
& \text { For } m=0, \ldots, M \\
& \text { 1. Sample the static paramaters from their prior } \\
& \quad \text { distribution, i.e. } \xi_{k}^{(m)}=\xi_{k-1}^{(m)} \\
& \text { 2. Update the predicted Kalman equations using (9) } \\
& \text { 3. Sample } \theta_{k}^{(m)} \text { from the optimal importance function } \\
& \text { using (18) } \\
& \text { 4. Update the filtered kalman equations using (10) } \\
& \text { 5. Evaluate the corresponding weights using (20) } \\
& \text { 6. Resampling step if } N_{e f f}<N / 2 \\
& \text { Evaluate the channel estimate } \widehat{\mathbf{h}} \text { using (21). }
\end{aligned}
$$




\section{SIMULATIONS}

In this section, the performance of the proposed MPF is studied through simulations. The following system parameters are assumed : a Rayleigh multipath channel with a delay of $L=10$ taps and a uniform power delay profile, an OFDM training symbol with $N=64$ subcarriers and a cyclic prefix of $N_{c p}=16$ samples. Each subcarrier is arbitrarily modulated in QPSK. The CFO $\epsilon$ is drawn for each OFDM symbol from a uniform distribution in $[-0.5 ; 0.5]$. The proposed marginalized particle filter is implemented with 100 particles.

Since no algorithms have been proposed to solve this problem without prior statistical knowledge of PHN and SNR, the mean square error (MSE) of the channel estimation is compared to the posterior Cramér-Rao Bound (CRB) for an OFDM channel estimator without PHN and CFO distortions. Since the DSS model (4) becomes linear and gaussian, it can be shown that the CRB is equal to the covariance of $p\left(\mathbf{h} \mid \theta_{0: k}, \xi_{0: k}, r_{0: k}\right)$ defined in (10) [16] :

$$
\operatorname{MSE}_{C R B}=\mathrm{E}\left[\operatorname{trace}\left[\Sigma_{N-1 \mid N-1}\right]\right]
$$

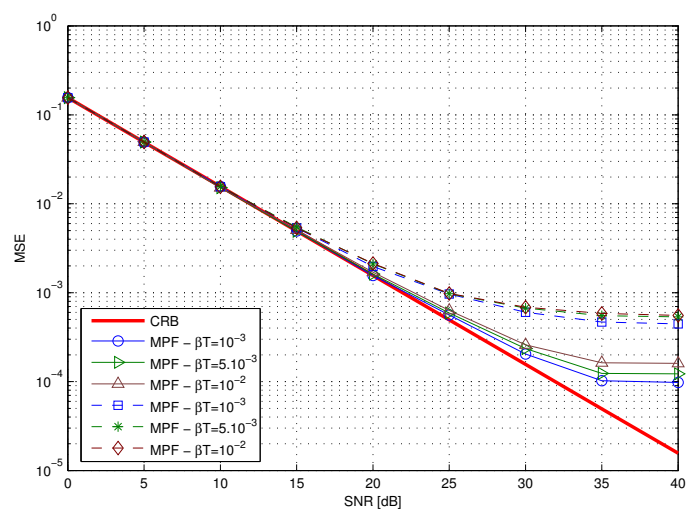

Fig. 1. MSE of channel estimation vs. SNR for different phase noise rates $\beta T$ with only $\sigma_{v}^{2}$ unknown (solid lines) or both $\sigma_{v}^{2}$ and $\sigma_{b}^{2}$ unknown (dashed lines).

Fig. 1 shows the channel estimation accuracy as a function of phase noise rate $\beta T$ and SNR. The MSE is obtained using :

$$
\operatorname{MSE}=\frac{1}{X} \sum_{n=1}^{X} \sum_{l=0}^{L-1}\left(h_{l}^{n}-\widehat{h}_{l}^{n}\right)^{2}
$$

where $X$ is the number of OFDM symbols used in simulations. The results demonstrate the robustness of the proposed MPF algorithm when the a priori statistics of the model are not perfectly known. As can be seen from this figure, for a SNR less than $15 \mathrm{~dB}$, the proposed MPF optimally estimates the CIR and leads to the same performances whatever the degree of assumption about prior statistics. CIR estimation performs better when only phase noise rate is unknown. For a large SNR, MSE curve tends towards a minimum MSE threshold depending on the phase noise rate. This is due to the fact that the covariance matrix of each Kalman filter doesn't depend on $\theta_{k}^{(j)}$ and $\epsilon_{k}^{(j)}$ and consequently the Kalman filter has the same convergence speed whatever phase noise rate.

\section{CONCLUSION}

In this paper, we consider the problem of OFDM channel estimation in the presence of CFO and PHN. Moreover, we study the difficult task where prior statistics of PHN and AWGN is assumed unknown at the receiver. To solve this problem, we propose an original marginalized particle filter based on the hybrid importance function. The use of this hybrid importance function is made possible by deriving an approximate optimal importance function for PHN. Numerical simulations demonstrate the effectiveness and the robustness of the proposed MPF algorithm for OFDM channel estimation in the presence of CFO and PHN without knowledge of prior statistical information. A such algorithm can be efficiently used for the design of OFDM receivers in wireline and wireless communication systems.

\section{REFERENCES}

[1] T. Pollet, M. Van Bladel, and M. Moeneclaey, "BER sensitivity of OFDM systems to carrier frequency offset and Wiener phase noise," IEEE Trans. Commun., vol. 43, pp. 191-193, Feb. 1995.

[2] L. Tomba, "On the effect of Wiener phase noise in OFDM systems," IEEE Trans. Commun., vol. 46, pp. 580-583, May 1998.

[3] C. Garnier, L. Clavier, Y. Delignon, M. Loosvelt, and D. Boulinguez, "Multiple access for $60 \mathrm{GHz}$ mobile ad hoc networks," in Proc. IEEE VTC Spring, May 2002, vol. 3, pp. $1517-1521$.

[4] S. Wu and Y. Bar-Ness, "A new phase noise mitigation method in OFDM systems with simultaneous CPE and ICI correction," in Proc. MCSS, Sept. 2003.

[5] D. Petrovic, W. Rave, and G. Fettweis, "Intercarrier interference due to phase noise in OFDM - estimation and suppression," in Proc. IEEE VTC Fall, Sept. 2004.

[6] D. D. Lin and T. J. Lim, "The variational inference approach to joint data detection and phase noise estimation in OFDM," IEEE Trans. Signal Process., to appear.

[7] K. Nikitopoulos and A. Polydoros, "Compensation schemes for phase noise and residual frequency offset in OFDM systems," in Proc. IEEE GlobeCom'01, Nov. 2002, vol. 1, pp. 330 - 333.

[8] S. Wu and Y. Bar-Ness, "OFDM channel estimation in the presence of frequency offset and phase noise," in Proc. IEEE ICC, May 2003, vol. 5, pp. 3366-3370.

[9] D.D. Lin, R.A. Pacheco, T.J. Lim, and D. Hatzinakos, "Joint estimation of channel response, frequency offset and phase noise in OFDM," IEEE Trans. Signal Process., vol. 54, no. 9, pp. 3542-3554, Sept. 2006.

[10] A. Doucet, N. De Freitas, and N. Gordon, Sequential Monte Carlo Methods in Practice, Springer-Verlag, 2001.

[11] J.S. Liu and R. Chen, "Sequential monte carlo methods for dynamic systems," Journal of the American Statistical Association, vol. 93, no. 443, pp. 1032-1044, 1998.

[12] M. Bolic, P.M. Djuric, and Sangjin Hong, "New resampling algorithms for particle filters," in Proc. IEEE ICASSP, April 2003.

[13] R. Chen and J. S. Liu, "Mixture kalman filters," Journal of the Royal Statistical Society, vol. 62, pp. 493 - 508, 2000.

[14] T. Schon, F. Gustafsson, and P.J. Nordlund, "Marginalized particle filters for mixed linear/nonlinear state-space models," IEEE Trans. Signal Process., vol. 53, pp. 2279 - 2289, July 2005.

[15] Y. Huang and P.M. Djuric, "A hybrid importance function for particle filtering," IEEE Signal Process. Lett., vol. 11, no. 3, pp. 404-406, March 2004.

[16] P. Tichavsky, C.H. Muravchik, and A. Nehorai, "Posterior cramér-rao bounds for discrete-time nonlinear filtering," IEEE Transactions on Signal Processing, vol. 46, no. 5, pp. 1386 - 1396, May 1998. 